\title{
Author Correction: Biallelic mutations in SORD cause a common and potentially treatable hereditary neuropathy with implications for diabetes
}

Andrea Cortese (D), Yi Zhu, Adriana P. Rebelo (D), Sara Negri, Steve Courel, Lisa Abreu, Chelsea J. Bacon, Yunhong Bai, Dana M. Bis-Brewer, Enrico Bugiardini, Elena Buglo, Matt C. Danzi, Shawna M. E. Feely, Alkyoni Athanasiou-Fragkouli, Nourelhoda A. Haridy, Inherited Neuropathy Consortium, Rosario Isasi, Alaa Khan, Matilde Laurà, Stefania Magri, Menelaos Pipis, Chiara Pisciotta, Eric Powell, Alexander M. Rossor, Paola Saveri, Janet E. Sowden, Stefano Tozza, Jana Vandrovcova, Julia Dallman, Elena Grignani, Enrico Marchioni, Steven S. Scherer, Beisha Tang, Zhiqiang Lin, Abdullah Al-Ajmi, Rebecca Schüle, Matthis Synofzik, Thierry Maisonobe, Tanya Stojkovic, Michaela Auer-Grumbach, Mohamed A. Abdelhamed, Sherifa A. Hamed, Ruxu Zhang, Fiore Manganelli, Lucio Santoro, Franco Taroni, Davide Pareyson, Henry Houlden, David N. Herrmann, Mary M. Reilly, Michael E. Shy, R. Grace Zhai (D) and Stephan Zuchner (D)

Correction to: Nature Genetics https://doi.org/10.1038/s41588-020-0615-4, published online 4 May 2020.

In the version of this article initially published, Rosemary R. Shy was not included as a member of the Inherited Neuropathy Consortium. The error has been corrected in the HTML and PDF versions of the article. 